Supplement of Earth Syst. Sci. Data, 11, 1153-1170, 2019

Earth System

https://doi.org/10.5194/essd-11-1153-2019-supplement

(C) Author(s) 2019. This work is distributed under

the Creative Commons Attribution 4.0 License.

(c) (1)

Science

亏Data

Supplement of

\title{
GRACE-REC: a reconstruction of climate-driven water storage changes over the last century
}

Vincent Humphrey and Lukas Gudmundsson

Correspondence to: Vincent Humphrey (vincent.humphrey@bluewin.ch)

The copyright of individual parts of the supplement might differ from the CC BY 4.0 License. 


\section{Contents}

- Supplementary Text S1

- $\quad$ Supplementary Figures S1-S4

\section{Supplementary Text S1 - Derivation of the TWS state at equilibrium (Eq. 5 of main manuscript)}

In the main manuscript, the equation representing the TWS response to temporal changes in precipitation

10 and residence time (Eq. 1) is the following

$$
\operatorname{TWS}(t)=(\operatorname{TWS}(t-1)) \cdot \mathrm{e}^{-\frac{1}{\tau(t)}}+P(t)
$$

where $t$ is a daily time vector, $\operatorname{TWS}(t)$ is the storage, $P(t)$ is the precipitation input and $\tau(t)$ is the

residence time of the water store. Also consider that $\mathrm{e}^{-\frac{1}{\tau(\mathrm{t})}}$ is always larger than 0 and smaller than 1 in 15 our application (as the residence time $\tau$ is at least larger than zero and not infinite).

This function requires to arbitrarily choose the state of TWS at the start of the simulation (i.e. the initial condition of TWS at $\mathrm{t}=0$ ). If storage is assumed to be empty (i.e. $\operatorname{TWS}(0)=0$ ), a spin-up period is necessary before the water store saturates around some average equilibrium value (Figure S1a). In previous work, the first 6 years of simulation had to be discarded because of this spin-up period (Humphrey et al., 2017). 
Here, our goal is to estimate an equilibrium value for TWS which we can use as a more realistic initial condition than $\operatorname{TWS}(0)=0$.

As a first approximation, let us reformulate equation (1) with the assumption of a mean precipitation input and a mean residence time.

5

$$
\begin{aligned}
& p=\operatorname{Mean}(P(t)) \\
& k=\operatorname{Mean}\left(\mathrm{e}^{-\frac{1}{\tau(\mathrm{t})}}\right) \\
& \operatorname{TWS}(\mathrm{t})=(\operatorname{TWS}(t-1)) \cdot k+p
\end{aligned}
$$

10 The result of this approximation can be seen in Figure S1b, and the equilibrium value obtained at the end of this simulation is $88.55 \mathrm{~mm}$, which is very close to the average TWS value of Figure S1a $(88.97 \mathrm{~mm}$ over the period 1984-2016).

In order to obtain a more practical analytical solution to equation (4), the iterative equation can be reformulated as a first-order linear ordinary differential equation:

15

$$
\frac{d \mathrm{TWS}}{d t}=-(1-k) \mathrm{TWS}+p
$$

The solution of which is:

$$
\mathrm{TWS}=c_{1} e^{-(1-k) t}+\frac{p}{1-k}
$$

Because $k$ is always between 0 and 1 as mentioned earlier, the equilibrium value of TWS when $t \rightarrow \infty$ (called $\mathrm{TWS}_{0}$ ), is simply the right-hand term of Eq. (6):

$$
\mathrm{TWS}_{0}=\frac{p}{1-k}
$$

25

Which corresponds to equation 5 of the main manuscript. 

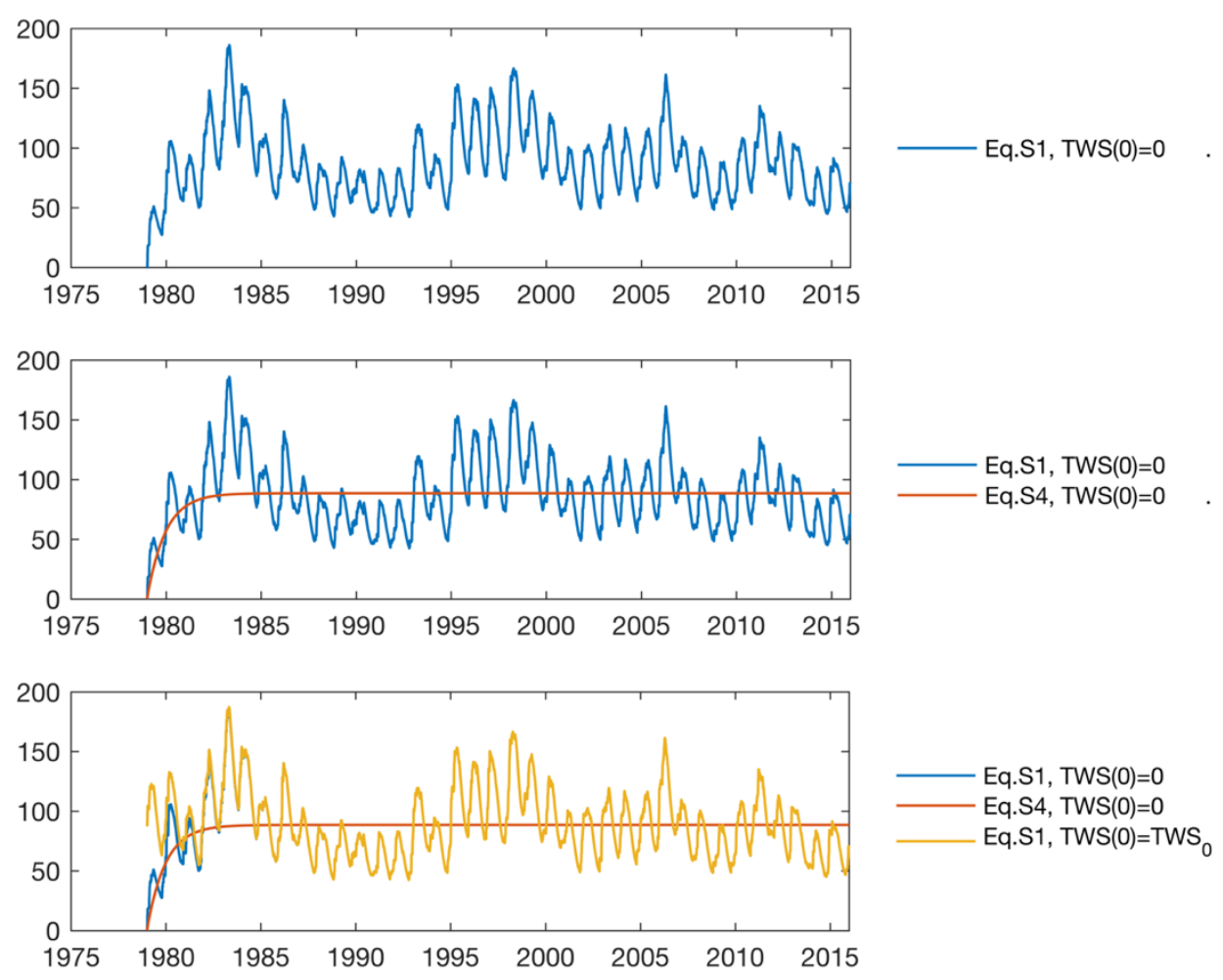

5 Figure S1. Same illustrative data as the one used in Figure 1 of the main manuscript, in units of mm TWS.

a) resulting TWS variability when using Equation (1) with $\operatorname{TWS}(0)=0$ as initial condition. The spin-up period can clearly be seen at the beginning of the time series. b) estimation of the equilibrium TWS based on Equation (4). c) resulting TWS variability when using Equation (1) with $\operatorname{TWS}(0)=\mathrm{TWS}_{0}$ as initial condition (i.e. using the equilibrium TWS as initial condition). 

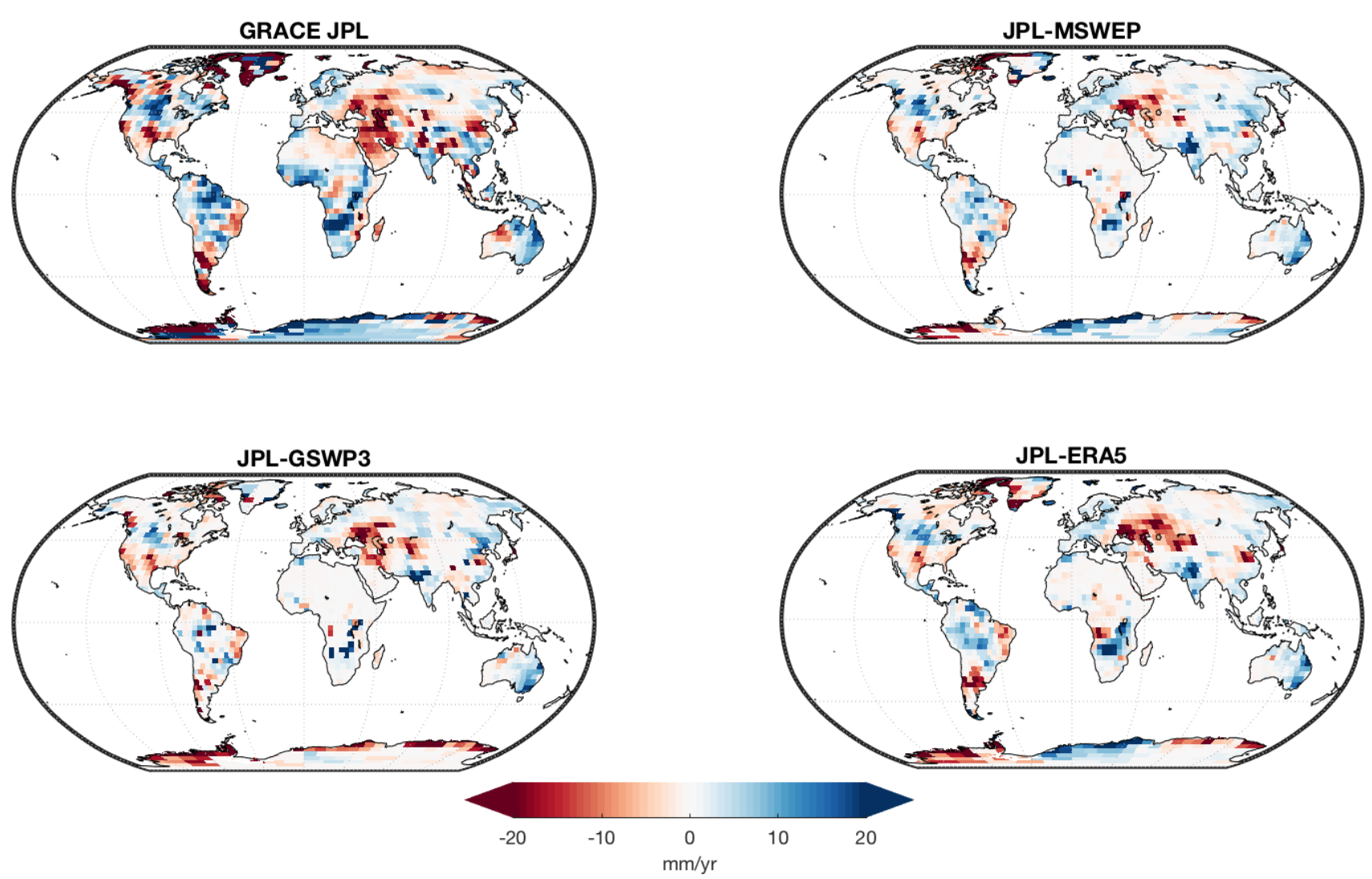

Figure S2. Linear trends obtained over the period 2003-2014 for the GRACE JPL Mascons and for the three associated reconstructions. 

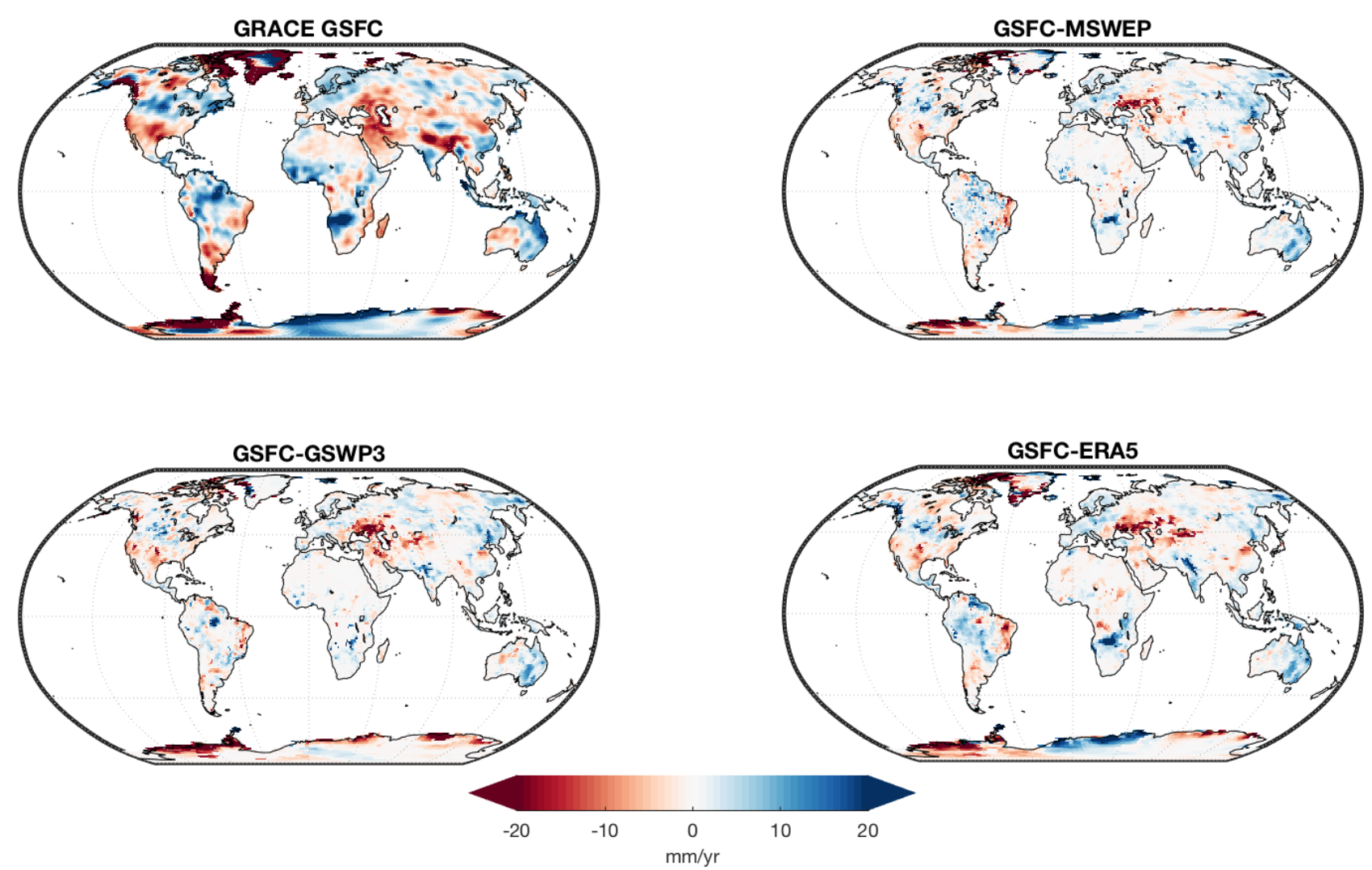

Figure S3. Linear trends obtained over the period 2003-2014 for the GRACE GSFC Mascons and for the three associated reconstructions. 

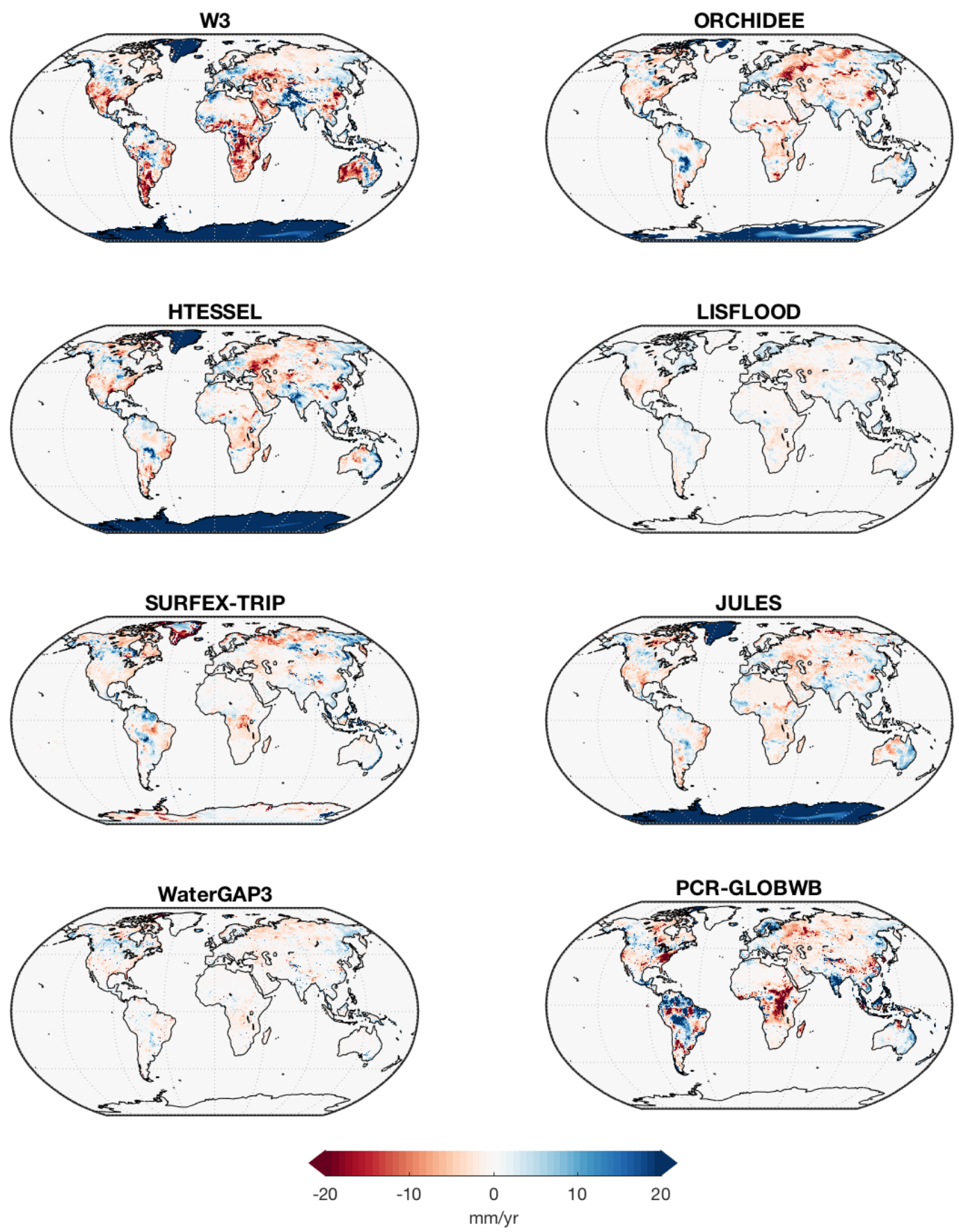

Figure S4. Linear trends obtained over the period 2003-2014 for the 8 WRR2 models. 